\title{
NUEVOS REGISTROS DE DISTRIBUCIÓN DE CUATRO ESPECIES DE SALAMANDRAS (CAUDATA: PLETHODONTIDAE) EN LA REGIÓN DE LAS ALTAS MONTAÑAS, VERACRUZ, MÉXICO.
}

\section{NEW DISTRIBUTION RECORDS OF FOUR SALAMANDERS SPECIES (CAUDATA: PLETHODONTIDAE) IN THE HIGH MOUNTAIN REGION, VERACRUZ, MEXICO.}

\author{
Ángel Iván Contreras Calvario ${ }^{1 *}$, Jorge luis Castillo Juárez ${ }^{1}$, Rene Avalos Vela ${ }^{1}$, Nelson M. \\ CERÓN DE LA LUZ ${ }^{2}$ Y ABIGAIL MORA REYES ${ }^{1}$ \\ ${ }^{1}$ Universidad Veracruzana, Facultad de Ciencias Biológicas y Agropecuarias, camino viejo Peñuela-Amatlán de los Reyes. S/n. 94945. Mpio. Amatlán \\ de los Reyes, Veracruz, México. \\ ${ }^{2}$ Colegio de post graduados, posgrado en Recursos genéticos y productividad, Carretera México-Texcoco km. 36.5 Montecillo, Texcoco, Estado de México \\ C.P. 56230, México. \\ "Correspondence: acontrerascalvario@gmail.com
}

Abstract.- Fourteen records of four species of the Plethodontidae family (Parvimolge townsendi, Pseudoeurycea firscheini, Thorius pennatulus and Aquiloeurycea cafetalera) are provided, eleven of these are new locations. The set of records is in nine municipalities located in the western center of the state of Veracruz (High Mountains Region), México.

Keywords.- Amphibians, biogeography, Canyon of the Río Blanco, lungless salamanders.

Resumen.- Se aportan catorce registros de cuatro especies de la familia Plethodontidae (Parvimolge townsendi, Pseudoeurycea firscheini, Thorius pennatulus y Aquiloeurycea cafetalera), once de estos son nuevas localidades. El conjunto de registros se encuentra en nueve municipios ubicados en el centro occidente del estado de Veracruz (Región de las Altas Montañas), México.

Palabras clave.- Anfibios, biogeografía, Cañón del Río Blanco, salamandras apulmonadas.

Gran parte de la diversidad de anfibios en México está representada por la familia Plethodontidae, con 132 especies destacando los géneros Chiropterotriton (18 spp), Ixalotriton (2 spp), Parvimolge (1 sp) y Thorius (29 spp) ya que estos son totalmente endémicos para el país, al igual que los más recientes géneros, Aquiloeurycea (6 spp) e Isthmura (7 spp), estos dos últimos distribuidos principalmente en la Sierra Madre Oriental, el Eje Volcánico Transversal y las tierras altas del sur de México (ParraOlea et al., 2014; Rovito et al., 2015; Sandoval-Comte et al., 2017; AmphibiaWeb, 2019)

Pseudoeurycea firscheini. En referencia a sus caracteres morfológicos estas salamandras presentan 13 pliegues costales, nostrilos de tamaño pequeño, surcos nasolabiales finos y presenta un surco en las esquinas de la boca que se extiende hasta el ojo (Shannon \& Werler, 1955).

$\mathrm{Su}$ distribución es conocida a una altitud de 1600 a 2200 msnm. Habita bosques de pino-encino, así como bosque nuboso (pudiéndole encontrar en los bordes) y parece requerir la presencia de abundantes bromelias y arboles maduros, no tolera hábitats muy degradados. Es una especie rara, pero se le localizada consistentemente en pequeños números en las localidades conocidas (amenazadas por el decline continuo en la 
extensión y calidad del hábitat) como en el área de Acultzingo (localidad tipo), Tequila, San Andrés Tenejapa en el centro-oeste de Veracruz y se sospecha de su ocurrencia en el Parque Nacional Cañón del Río Blanco (IUCN, 2016; Frost, 2018; Kelly-Hernández et al., 2019).

Parvimolge townsendi. En cuanto a sus características morfológicas, presenta de 4 a 5 pliegues costales, la forma de su cabeza es ovalada, en adultos los nostrilos son muy prominentes, la cola es grande semejante a la longitud de cuerpo y cabeza (Dunn, 1922).

Habita en la región montañosa del centro de Veracruz, en elevaciones intermedias entre 800 y $2000 \mathrm{msnm}$. en bosque nuboso y bosque semideciduo tropical, $87 \%$ de su distribución potencial coincide con la de T. pennatulus (Sandoval-Comte et al., 2012). Los municipios mencionados en este trabajo no se señalaron con anterioridad, pues esta especie se ha reportado para Ixtaczoquitlan, Huatusco de Chicuellar, Fortín de las Flores y Xalapa, en el estado de Veracruz, México (Vernet, 2018).

Thorius pennatulus. En cuanto a características morfológicas más relevantes de esta especie es que posee una cabeza relativamente ancha, el hocico corto y con una terminación puntiaguda, presenta fosas nasales pequeñas más prominentes en los machos, no presenta dientes maxilares, posee 13 pliegues costales y presentan una cola robusta relativamente al grosor del cuerpo (Cope, 1869; Hanken \& Wake, 1998).

T. pennatulus está preferentemente asociado al bosque nuboso (entre los 800 y $2000 \mathrm{msnm}$ ) aunque también se ha reportado en bosque tropical semideciduo y su distribución potencial coincide en un $75 \%$ con la de P. townsendi. En la parte central de Veracruz dichas distribuciones se encuentran ampliamente fragmentadas y restringidas (Sandoval-Comte et al., 2012).

Aquiloeurycea cafetalera. Fue recientemente descrita, se trata de una salamandra de tamaño mediano, la especie presenta en la región dorsal color chocolate oscuro en la cabeza y el resto del dorso es color marrón, manchones en el hocico color gris, el vientre es de un tono marrón oscuro con pequeñas manchas grises (esto puede variar presentando el dorso una coloración marrón claro en la cabeza y marrón rojizo en el resto del cuerpo y en la región ventral desde la región gular, las extremidades y la cola un color gris claro). En cuanto a sus características morfológicas esta especie tiene una cabeza aplanada y boca redondeada, el cuello es ancho al igual que su cabeza, presenta 13 surcos costales, una cola prominente y gruesa (Parra-Olea et al., 2010; Aguilar-López et al., 2017).

A. cafetalera se conoce en un rango entre 1250 y $2220 \mathrm{msnm}$ en bosque nuboso y plantaciones de café de sombra con bosques adyacentes, desde las cercanías de la localidad tipo en Huatusco y al norte de La Joya, también cerca de la localidad de Zoncolco (se presume su presencia en bosques nubosos entre estas localidades), en tanto que su distribución más septentrional es el municipio de Atzalan, esto en el estado de Veracruz, mientras que para Puebla se conoce en el municipio de Chichiquila. Se cree que no se encuentra en el Cerro Chicahuaxtla y otros sitios bien recolectados cerca de la ciudad de Córdoba, Ver. esto pese a que parecen figurar en la distribución potencial más reciente para la especie (Parra-Olea et al., 2010; Aguilar-López et al., 2017).

Para el presente trabajo realizamos múltiples expediciones de campo en donde se buscó de manera directa las distintas especies de salamandras expuestas en este trabajo. Documentamos los registros fotográficamente y tomamos los datos GPS correspondientes a cada organismo de su respectiva localidad.

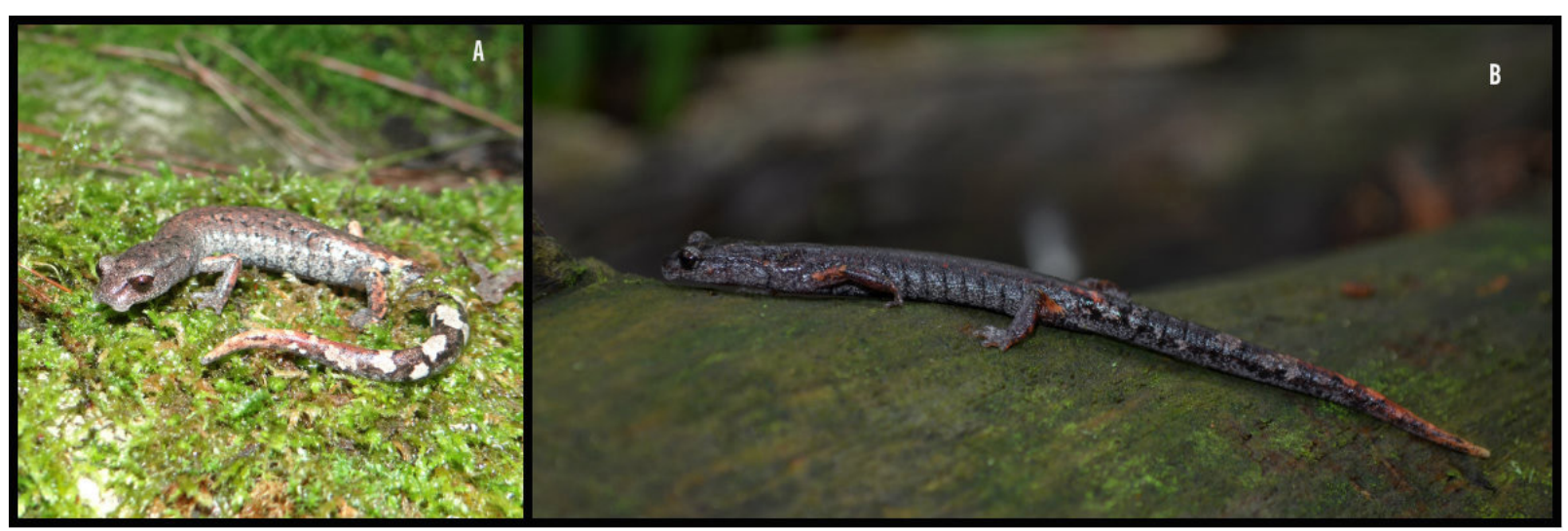

Figure 1. Individuals of Pseudoeurycea firscheini in localities of Veracruz, México. Photographs by Contreras Calvario (A), Avalos Vela (B).

Figura 1. Individuos de Pseudoeurycea firscheini en localidades de Veracruz, México. Fotografías por Contreras Calvario (A), Avalos Vela (B). 
Realizamos un mapa con los nuevos registros y el registro conocido más cercano en la región de cada una de estas especies utilizando el software QGIS versión 2.18.

Nuevas localidades:

\section{Pseudoeurycea firscheini (Shannon \& Werler, 1955)}

- México, Veracruz, municipio de Camerino Z. Mendoza, Necoxtla, (18 ${ }^{\circ} 46^{\prime} 59.68^{\prime \prime} \mathrm{N} 97^{\circ}$ o9'16.80 "O; WGS 84) 2114 m, 20 agosto 2016. Observamos un ejemplar bajo una roca en un fragmento de bosque de pino-encino al lado de un camino de terracería. (Fig. 1A).

- México, Veracruz, municipio de Camerino Z. Mendoza, Necoxtla, (18 $46^{\prime} 58.52^{\prime \prime} \mathrm{N} 97^{\circ}$ o9'14.22"O; WGS 84) $2134 \mathrm{~m}, 05$ agosto 2018. Hallamos tres salamandras en un bosque de pino encino, en tres microhábitats diferentes (bajo roca, bajo tronco caído y bajo musgo), muy cercanas entre sí. (Fig. 1B).

-- México, Veracruz, Municipio de Nogales, Palo Verde (18 ${ }^{\circ} 49^{\prime} 33.54^{\prime \prime} \mathrm{N} 97^{\circ} 10^{\prime} 17.89^{\prime \prime} \mathrm{O}$; WGS 84). $1418 \mathrm{~m}$, o9 agosto 2018. Encontramos un ejemplar bajo una roca a la orilla del camino, en donde se presenta vegetación secundaria. La vegetación original circundante es bosque mesófilo de montaña.

Los nuevos registros en este trabajo son los primeros para los municipios mencionados y llenan un vacío de distribución, pues estos se presentan al centro de su distribución conocida entre los municipios de Acultzingo (Shannon y Werler, 1955) y Tequila (Frost, 2018).

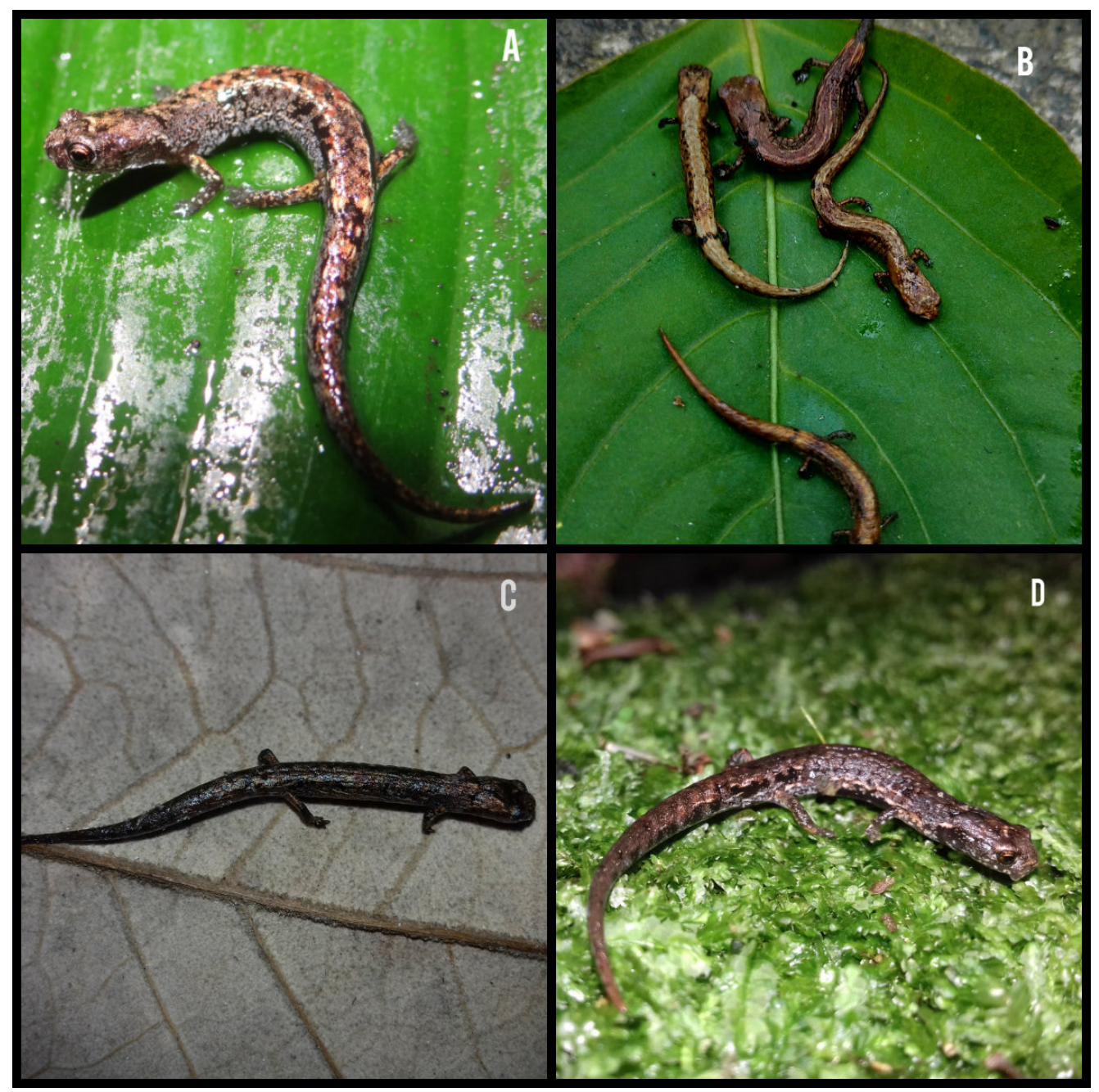

Figure 2. Individuals of Parvimolge townsendi in localities of Veracruz, México. Photographs by Castillo Juárez (B), Contreras Calvario (A, C, D).

Figura 2. Individuos de Parvimolge townsendi en localidades de Veracruz, México. Fotografías por Castillo Juárez (B), Contreras Calvario (A, C, D). 


\section{Parvimolge townsendi (Dunn, 1922)}

--México, Veracruz, Municipio de Chocaman, Tepexilotla. $\left(18^{\circ} 58^{\prime} 53.85^{\prime \prime} \mathrm{N}, 97^{\circ} 04^{\prime} 51.84^{\prime \prime} \mathrm{O}\right.$; WGS 84$) 1440 \mathrm{msnm}, 21$ enero 2018. Encontramos cuatro individuos bajo una roca a la orilla de un camino de terracería, un ejemplar con la cola incompleta. El tipo de vegetación presente es bosque mesófilo de montaña. La fotografía fue depositada en la Colección Nacional de Anfibios y Reptiles de la Universidad Nacional Autónoma de México (IBHRF-461), (Fig. 2A y B).

--México, Veracruz, Municipio de Chocaman, Tepexilotla. (18 ${ }^{\circ} 58^{\prime} 58.45^{\prime \prime} \mathrm{N}, 97^{\circ} 05^{\prime} 28.64^{\prime \prime} \mathrm{O}$; WGS 84) $1508 \mathrm{msnm}, 9$ abril 2018. Hallamos al ejemplar aproximadamente a 1 metro del río Metlác oculto entre un par de rocas, el sustrato consistía en arena proveniente del río, el tipo de vegetación que predomina en la zona es bosque mesófilo de montaña y un bosque de galería a lo largo del cauce del mencionado rio (Fig. 2C).

-- México, Veracruz, Municipio de Atzacan, El Nogal. ( $18^{\circ} 58^{\prime} 59.673^{\prime \prime} \mathrm{N} 97^{\circ} 05^{\prime} 38.84^{\prime \prime} \mathrm{O}$ WGS 84) $1670 \mathrm{msnm}, 10$ marzo 2018. Encontramos una salamandra bajo una roca a la orilla de un arroyo intermitente, en un pequeño parche de bosque mesófilo de montaña cuya vegetación circundante es un potrero. La fotografía fue depositada en la Colección Nacional de Anfibios y Reptiles de la Universidad Nacional Autónoma de México (IBHRF-462), (Fig. 2D).

--México, Veracruz: Municipio de Orizaba, Parque Ecológico Cerro del Borrego ( $18^{\circ} 50^{\prime} 46.78^{\prime \prime} \mathrm{N} 97^{\circ} 07^{\prime} 00.52^{\prime \prime O}$ WGS 84) 1400 $\mathrm{m}, 16$ de enero de 2019. Localizamos tres ejemplares debajo de troncos en descomposición, la vegetación consta de bosque mesófilo de montaña.

Estos recientes hallazgos se encuentran cerca de las localidades históricas y dentro del modelo de distribución potencial propuesto por Sandoval-Comte et al. (2012), quién señala que la búsqueda de ejemplares se debe realizar en zonas donde no se conoce la diversidad de la herpetofauna, por lo que se infiere que los nuevos registros en la región son relevantes pues sumado a los registros conocidos completan un hueco en la distribución entre las localidades de la región capital de Veracruz, Pájaro Verde en Puebla y Santa María Chilchotla en el estado de Oaxaca (CONABIO, s/f).

\section{Thorius pennatulus (Cope, 1869)}

--México: Veracruz, Municipio de Chocaman, Tepexilotla (18 $58^{\prime} 53.13^{\prime \prime} \mathrm{N}, 97^{\circ} 05^{\prime} 03.01^{\prime \prime} \mathrm{O}$; WGS 84) $1511 \mathrm{~m}, 21$ enero 2018. Hallamos un organismo bajo una roca a la orilla de un camino de terracería transitado, el tipo de vegetación de la zona pertenece a bosque mesófilo de montaña (Fig. 3B).

--México: Veracruz Municipio de San Juan Texhuacan $\left(18^{\circ}\right.$ $35^{\prime} 47.63^{\prime \prime}$ N, 97 02'09.83"O; WGS 84) 1865 m, O3 marzo 2018. Encontramos un ejemplar bajo plantas epifitas del género Tillandsia que se encontraban en el suelo, la vegetación predominante en el lugar es bosque de encino-pino. (Fig. 3C).

Su distribución es conocida desde las montañas bajas en el centro-oeste de Veracruz, México. Desde Soledad Atzompa en el sur y oeste, al este hasta Cerro Chicahuaxtla y al norte hasta Teocelo (Frost, 2018). De esta forma con los datos de estas nuevas localidades contribuimos a llenar algunos vacíos en los registros

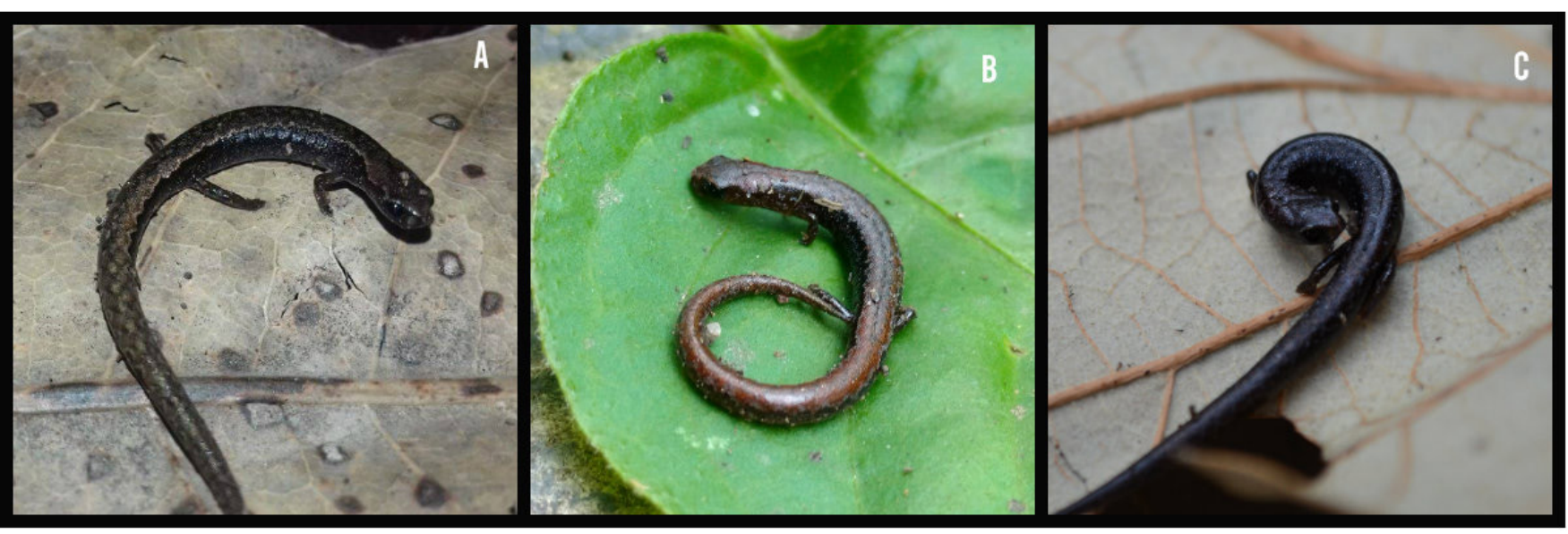

Figure 3. Individuals of Thorius pennatulus in localities of Veracruz, México. Photographs by Contreras Calvario (A), Castillo Juárez (B), Avalos Vela (C).

Figura 3. Individuos de Thorius pennatulus en localidades de Veracruz, México. Fotografías por Contreras Calvario (A), Castillo Juárez (B), Avalos Vela (C). 
de campo conocidos y ampliar el conocimiento de distribución de esta especie.

\section{Aquiloeurycea cafetalera (Parra-Olea, Rovito, Márquez-} Valdelmar, Cruz, Murrieta-Galindo \& Wake, 2010)

--México, Veracruz: Municipio de Zongolica, El Tenango (18 '39'57.06" N, $96^{\circ} 59^{\prime} 31.89$ " O; WGS 84) 1401 msnm, 12 de mayo de 2016. Encontramos la salamandra activa sobre un tronco caído aproximadamente a las 22:00 h, en un bosque nuboso donde la vegetación consiste principalmente en árboles del género Liquidambar, Quercus y Cupressus.

--México, Veracruz, Municipio de Chocaman, (1858'54.94" N, 97०05'23.29" O; WGS 84) $1502 \mathrm{~m}, 7$ de abril del 2018. Encontramos el ejemplar entre la hojarasca, aproximadamente 1 metro a la orilla del rio Metlác. El tipo de vegetación predominante en la zona corresponde a bosque mesófilo de montaña sin embargo la orilla del río donde se halló el organismo es recorrida por un bosque de galería de la especie Platanus mexicana Moric, cercano a un asentamiento rural (Fig. 4B).

Precisamente como lo indica Aguilar-López et al. (2017) solo el trabajo de campo puede acreditar los modelos potenciales. Por esto, nuestros registros sumados a las observaciones de sus recientes localidades y las localidades históricas representan la distribución real conocida para la especie hasta el momento.

Partiendo del hecho que $P$. firscheini, se encuentra incluida en la Lista Roja de la Unión Internacional para la Conservación de la Naturaleza en la categoría de "En Peligro de Extinción" (EN) y en la NOM-059 como sujeta a Protección Especial (Pr), P. townsendi en "Peligro Crítico" (CR) en la IUCN y como Amenazada en la NOM-059 y T. pennatulus en Peligro Crítico en la IUCN y sujeta a Protección Especial en la NOM-059 (Parra-Olea et al., 2008; Parra-Olea et al., 2008b; SEMARNAT, 2010; IUCN, 2016). Al igual que la más reciente evaluación de A. cafetalera sugiere que conforme a los criterios propuestos por la IUCN y SEMARNAT esta se puede considerar como "Vulnerable" (VU) y ubicar bajo la categoría de amenazada (A) respectivamente (Aguilar-López et al., 2017), consideramos que, al estar sus hábitats en constante destrucción y modificación por actividades antropogénicas, cada nuevo registro aporta datos importantes para tomar acciones respecto a la conservación de estas especies y conocer más sobre su historia natural y distribución real, más allá de los modelos potenciales.

Además de los 11 nuevos registros, durante nuestro trabajo de campo reportamos tres localidades más:

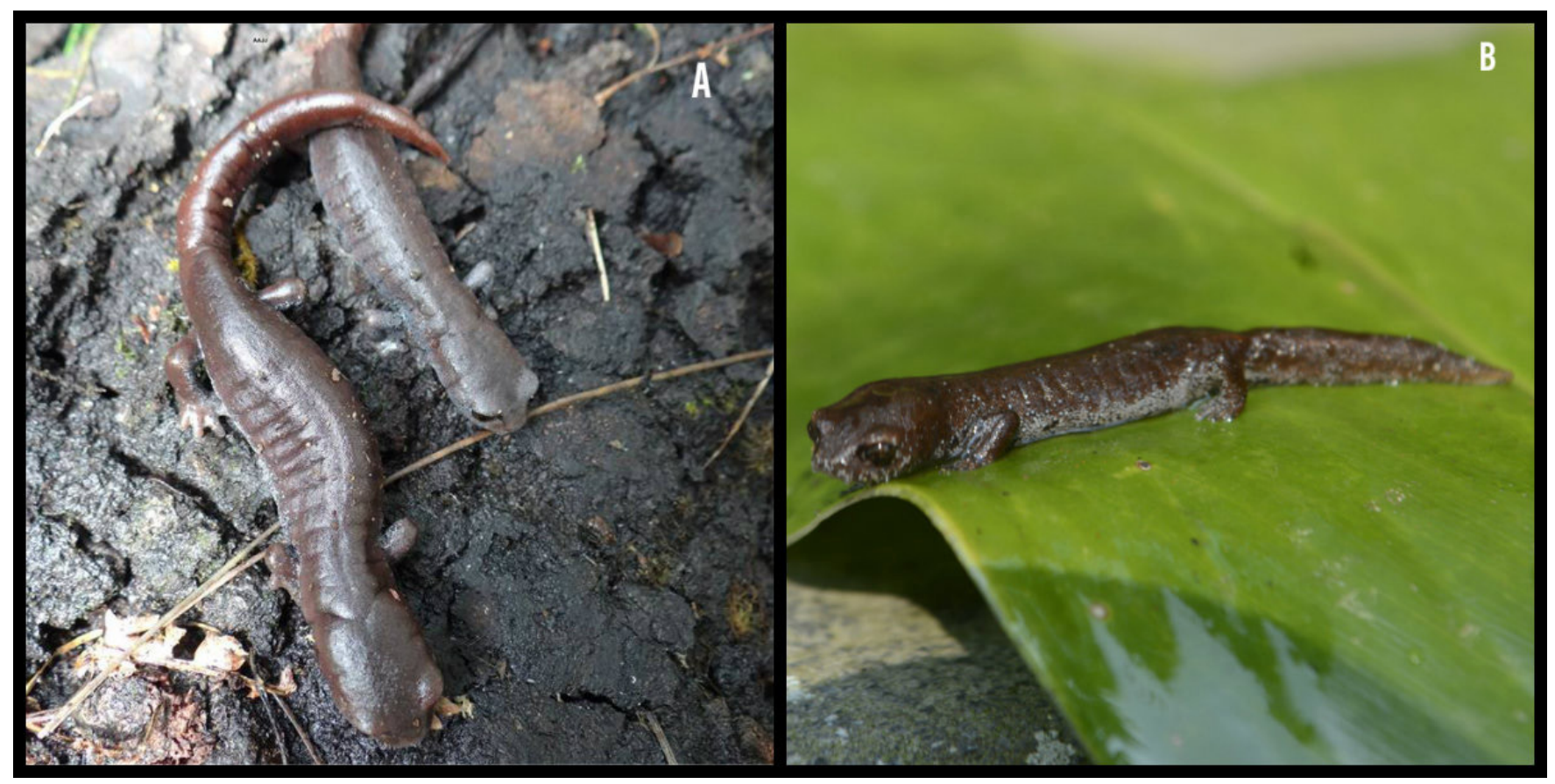

Figure 4. Individuals of Aquiloeurycea cafetalera in localities of Veracruz, México. Photographs by Contreras Calvario (A), Castillo Juárez (B).

Figura 4. Individuos de Aquiloeurycea cafetalera en localidades de Veracruz, México. Fotografías por Contreras Calvario (A), Castillo Juárez (B). 


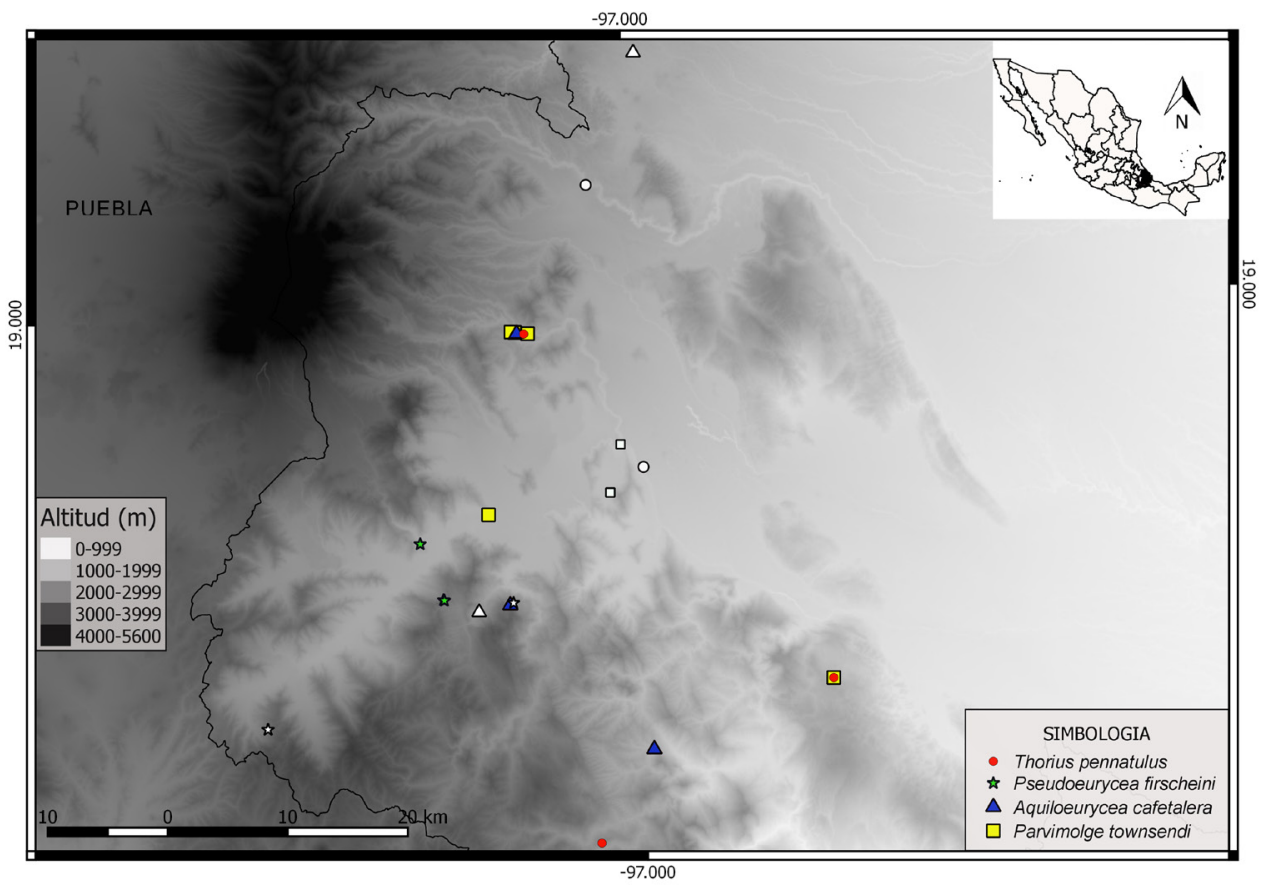

Figure 5. New localities of P. firscheini, P. townsendi, T. pennatulus and A. cafetalera in the Altas Montañas region, Veracruz, Mexico. Each species with its respective symbol, the symbols in white color represent the nearest historical localities.

Figura 5. Nuevas localidades de P. firscheini, P. townsendi, T. pennatulus y A. cafetalera en la región de las Altas Montañas, Veracruz, México. Cada especie con su respectivo símbolo, los símbolos en color blanco representan las localidades históricas más cercanas.

-- México, Veracruz, Municipio de Tezonapa, Colonia Agrícola Rincón de las Flores, (1842'53.64"N, 96 $50^{\circ} 54.49^{\prime \prime O}$; WGS 84) 1134 msnm, 22 junio 2017. La fotografía fue depositada en la Colección Nacional de Anfibios y Reptiles de la Universidad Nacional Autónoma de México (IBH-RF-460), (P. townsendi, Fig. 2A).

--México: Veracruz Municipio de Tezonapa, Colonia Agrícola Rincón de las Flores (18²4'53.64"N, 96 $50^{\circ}$ '54.49"W; WGS 84) 1134 msnm, 17 junio 2017. (T. pennatulus, Fig. 3A).

--México, Veracruz: Municipio de San Andrés Tenejapan, Cerro Petlalcala ( $\left.18^{\circ} 46^{\prime} 40.009^{\prime \prime} \mathrm{N}, 97^{\circ} 6^{\prime} 7.482^{\prime \prime} \mathrm{O}\right) 2120 \mathrm{msnm}, 24 \mathrm{de}$ noviembre de 2018 (A. cafetalera, Fig. 4A).

Anteriormente estos municipios fueron reportados por Vásquez-Cruz (2015) y Kelly-Hernández (2017) respectivamente y pese a no ser nuevos registros, confirmamos la permanencia en dichas localidades a pesar de la presión antropogénica que enfrentan estas especies, pues fueron halladas dos años después (Tezonapa) y un año después (San Andrés Tenejapan).

Con esta información se aportan 14 registros en nueve municipios, 11 de ellos nuevos y 3 más los consideramos de importancia para el conocimiento de estas salamandras en la región (Fig. 5) ubicados en el centro occidente del estado de Veracruz, México (denominada Región de las Altas Montañas) para estas cuatro especies de las cuales se conocía muy poco de su distribución hasta años recientes. Se remarcan el registro de menor altitud conocido (1418 msnm) y ocurrencia en vegetación secundaria para P. firscheini, así como su presencia a lo largo del polígono del Parque Nacional Cañón del Río Blanco. De igual manera la ocurrencia de $P$. townsendi y A. cafetalera en un bosque de galería (predominante de Platanus mexicana Moric) y de T. pennatulus en bosque de encino-pino.

Agradecimientos.- Agradecemos a Laura Pamela Taval Velázquez, Steven Bol, Magdaleno Domínguez Gálvez y a Ángel Ramírez Hernández por la colaboración en campo.

\section{LITERATURA CITADA}

Aguilar-López, J.L., A. Sandoval-Comte \& E. Pineda. 2017. Distribution, encounter rate and conservation status of Aquiloeurycea cafetalera (Caudata: Plethodontidae), a recently described Mexican salamander. Phyllomedusa: Journal of Herpetology 16(2):211-224. 
AmphibiaWeb: Information on amphibian biology and conservation. 2019. Berkeley, California: AmphibiaWeb. Disponible: http:// amphibiaweb.org/. [Consultado en septiembre 2019]

CONABIO. s/f. Enciclovida Salamandra enana de la Sierra Madre Oriental (Parvimolge townsendi). http://enciclovida. $\mathrm{mx} /$ especies/35278-parvimolge-townsendi. [Consultado en noviembre 2018]

Cope, E.D. 1869. A review of the species of the Plethodontidae and Desmognathidae. Proceedings of the Academy of Natural Sciences of Philadelphia 93-118.

Dunn, E.R. 1922. A new salamander from México. Biological Society of Washington 35:5-6.

Frost, D.R. 2018. Amphibian Species of the World: an Online Reference. Version 6.0. http://research.amnh.org/vz/ herpetology/amphibia/index.php. [Consultado en noviembre 2018]

Hanken, J. \& D. B. Wake. 1998. Biology of tiny animals: systematics of the minute salamanders (Thorius: Plethodontidae) from Veracruz and Puebla, Mexico, with descriptions of five new species. Copeia 1998(2):312-345.

IUCN SSC Amphibian Specialist Group. 2016. Pseudoeurycea firscheini. The IUCN Red List of Threatened Species 2016. http:// dx.doi.org/10.2305/IUCN.UK.2016-3.RLTS.T59377A53981942.en. [Consultado en noviembre 2018]

Kelly-Hernández, A. 2017. Actualización de la herpetofauna del Cerro Petlalcala, municipio de San Andrés Tenejapan, Veracruz, México. Tesis de licenciatura. Universidad Veracruzana, Amatlán de los Reyes, Veracruz, México.

Kelly-Hernández, A., S.M. Rovito \& V. Vásquez-Cruz. 2019. A new population of the endangered salamander Pseudoeurycea firscheini (Caudata: Plethodontidae) from Mexico, with notes on natural history. Phyllomedusa: Journal of Herpetology 18(1):97-100.

National Science Foundation. 2018. VertNet. versión 2016-09-29. http://portal.vertnet.org/ search?q=specificepithet:townsendi+genus:parvimolge. [Consultado en noviembre 2018]

Parra-Olea, G., O. Flores-Villela \& C. Mendoza-Almeralla. 2014. Biodiversidad de anfibios en México. Revista Mexicana de Biodiversidad 85:460-466.
Parra-Olea, G., S.M. Rovito, L. Márquez-Valdelamar, G. Cruz, R. Murrieta-Galindo \& D. Wake. 2010. A new species of Pseudoeurycea from the cloud forest in Veracruz, México. Zootaxa 2725:57-68.

Parra-Olea, G., D. Wake, J. Hanken \& M. García-París. 2008. Parvimolge townsendi. The IUCN Red List of Threatened Species 2008. http://dx.doi.org/10.2305/IUCN.UK.2008.RLTS. T59328A11918563.en. [Consultado en noviembre 2018]

Parra-Olea, G., D. Wake, J. Raffaëlli \& J. Hanken. 2008. Thorius pennatulus. The IUCN Red List of Threatened Species 2008. http:// dx.doi.org/10.2305/IUCN.UK.2008.RLTS.T59424A11938766.en. [Consultado en noviembre 2018]

Rovito, S.M., G. Parra-Olea, E. Recuero \& D. Wake. 2015 Diversification and biogeographical history of Neotropical plethodontid salamanders. Zoological Journal of the Linnean Society 175(1):167-188.

Sandoval-Comte, A., E. Pineda \& J.L. Aguilar-López. 2012. In Search of Critically Endangered Species: The Current Situation of Two Tiny Salamander Species in the Neotropical Mountains of Mexico. PloS One 7(4):e34023.

Sandoval-Comte, A., E. Pineda, S.M. Rovito \& R. Luría-Manzano. 2017. A new species of Isthmura (Caudata: Plethodontidae) from the montane cloud forest of central Veracruz, Mexico. Zootaxa 4277(4):573-582.

Secretaría de Medio Ambiente y Recursos Naturales. 2010. Norma Oficial Mexicana. NOM-059-SEMARNAT-2010. Protección ambiental-especies nativas de México de flora y fauna silvestres -categorías de riesgo y especificaciones para su inclusión o cambio-lista de especies en riesgo. Diario oficial de la federación, D. F., México.

Shannon, F.A. \& J.E. Werler. 1955. Report on a small collection of amphibians from Veracruz, with a description of a new species of Pseudoeurycea. Herpetologica 11(2):81-85.

Vásquez-Cruz, V. 2015. Anfibios y reptiles de la Colonia Agrícola Rincón de las Flores, Tezonapa, Ver. Tesis de licenciatura. Universidad Veracruzana, Amatlán de los Reyes, Veracruz, México.

Wake, D.B. \& J.F. Lynch. 1976. The distribution, ecology, and evolutionary history of plethodontid salamanders in tropical America. Natural History Museum of Los Angeles County 25:165 . 